\title{
A Case Series Measuring Functional Outcomes in Adult Patients Who Underwent Hip Arthroplasty Using the Modified Trochanteric Slide Approach
}

\author{
Nicole Teresa C. Lukban, MD and Gregorio Marcelo S. Azores, MD \\ Section of Arthroplasty, Department of Orthopedics, Philippine General Hospital, University of the Philippines Manila
}

\begin{abstract}
Background. Different trochanteric osteotomies have been developed to aid in surgical exposure and proper removal and placement of arthroplasty components.
\end{abstract}

Objectives. The study aimed to measure functional outcomes of the modified trochanteric slide approach for both primary and revision hip arthroplasty with radiologic and clinical variables, identify preoperative indications for the approach and identify possible postoperative complications.

Methods. We conducted a retrospective case series of patients who underwent the modified trochanteric slide approach for hip arthroplasty at the orthopedic department of a tertiary hospital from 2012 to 2016 . We reviewed patient charts and radiographs. Descriptive statistics were used to analyze data.

Results. Out of nine patients screened, seven were included. The average post-op hip range of motion was 42 degrees abduction and 98 degrees flexion. The union rate was $36 \%$ and the non-union rate was $7 \%$.

Conclusion. The modified osteotomy is still recommended for difficult primaries and revisions to aid in exposure and hip biomechanics post-operatively.

Keywords: arthroplasty, hip joint, osteotomy

Paper presented in the Residents' research forum on November 2018, at the Philippine Orthopedic Association Annual Congress.

Corresponding author: Nicole Teresa C. Lukban, MD

Department of Orthopedics

Philippine General Hospital

University of the Philippines Manila

Taft Avenue, Ermita, Manila 1000, Philippines

Email: nicole.lukban@gmail.com

\section{INTRODUCTION}

Different trochanteric osteotomies have been described for both primary and revision hip arthroplasty, to increase the exposure of the surgical field to aid in proper removal and placement of instrumentation and components. ${ }^{1}$ The type of osteotomy ultimately depends on what needs to be exposed, the quality of the patient's proximal fémur, ${ }^{1}$ the difficulty of component removal, and the complexity of the reconstruction. ${ }^{2}$

The traditional transtrochanteric approach by Charnley was used for difficult primary total hip arthroplasty cases, such as developmental dysplasia, severe protrusio, previous peritrochanteric fracture, takedown of a previous hip fusion, severe heterotrophic ossification due to previous surgical procedures, and severe congenital varus deformity. ${ }^{3}$ A lateral approach to the hip is performed, fascia lata split, vastus lateralis tendon divided transversely, the posterior margin of the gluteus medius retracted and osteotomy performed just distal to the origin of the vastus lateralis. This approach gives better exposure to both acetabulum and femur to aid in the proper alignment of the prosthesis. ${ }^{4}$ However, it 
Functional Outcomes of Modified Trochanteric Slide

is associated with nonunion from $0.5-38 \%{ }^{5}$ of the time, trochanteric migration, and recurrent dislocation, ${ }^{2}$ with associated prolonged operative time and blood loss. ${ }^{5}$

A modification done by Glassman involves the preservation of the vastus lateralis attachment to the trochanteric fragment, stabilizing the trochanter by counteracting the pull of the abductors and avoiding proximal migration. ${ }^{6}$ This approach, known as the trochanteric slide, is indicated in the removal of a well-fixed proximally porous-coated component and removal of a failed endoprosthesis and gives sufficient exposure of the circumference of the acetabulum. ${ }^{1}$ Non-union rate was at $5-10 \%$ with fragment migration and wire breakage in seven patients. Two patients underwent reoperation for component migration and infection. ${ }^{6}$ There were no reports of dislocations or reattachment for painful non-union. A study by Bal et al. ${ }^{7}$ studied 73 trochanters, where 67 (92\%) had healed to the femur, 3 with the fibrous union in situ and the other 3 with the displacement of the trochanteric fragment in the lateral and anterior direction relative to the original trochanteric bed. They have shown that nonunion can result in persistent abductor weakness, pain, and dislocation of the hip. The reoperation rate was at $28 \%$ due to painful bursitis or from hardware fragmentation, which was addressed by hardware removal.

This approach was used on acetabular fractures, with improved exposure of the iliac wing and anterior column, to stabilize transverse, T-type, and both column fractures. ${ }^{8}$ Radiographic union was $100 \%$ at 5 months. Two patients had implants removed because of trochanteric bursitis, two patients had a subsequent total hip replacement and one patient had removal of heterotrophic ossification.

The trochanteric slide was modified by Gross using a lateral approach, with the osteotomy anterior to the insertion of the posterior capsule and external rotators on the greater trochanter. ${ }^{9}$ Preservation of the soft tissue proximally by the abductor muscles and distally by the vastus lateralis, stabilizes the structures that resist posterior dislocation of the hip, decreasing the incidence of non-union and migration. ${ }^{2}$ Goodman reported a lower dislocation rate of $3.3 \%$ in acetabular revisions as compared to a traditional slide at 14.4\%; this, however, was not statistically significant. ${ }^{9}$

Lakstein reported $87 \%$ union, $11 \%$ fibrous union, and only $3 \%$ of non-union, who were however asymptomatic. ${ }^{10}$ Eleven percent had trochanteric pain but only 2 patients had persistent pain beyond 6 months; the pain, however, was severe. The dislocation rate was only $5 \%$.

Currently, no local studies have been made to investigate the epidemiology of the use of the modified trochanteric slide and its functional outcome. This study aimed to provide preliminary data on the modified trochanteric slide approach within the local setting.

The study asked: What were the functional outcomes in adult patients who underwent hip arthroplasty using the modified trochanteric slide approach?
The primary objective of this study was to measure functional outcomes of patients who underwent hip arthroplasty using the modified trochanteric slide approach based on:

- dislocation and revision rates

- trochanteric migration

- radiologic union

- clinical hip function

- post-operative pain

The secondary objectives were:

1. To identify the preoperative indications for the approach for both primary and revision hip arthroplasty

2. To identify post-operative complications

\section{MATERIALS AND METHODS}

The retrospective case series was conducted at the Department of Orthopedics, Philippine General HospitalUniversity of the Philippines after approval by the ethics board. We screened patients from the Philippine General Hospital Arthroplasty Census from 2012 to 2016 who underwent the modified trochanteric slide approach, as described by Gross. ${ }^{9}$

Follow-up charts were reviewed by the principal investigator, and clinical hip function was quantified in terms of the range of motion, mode of ambulation, and post-operative pain.

Radiographs were reviewed by a radiologist and an orthopedic surgeon using photographs of the plates during the follow-up. Radiographs were labeled by the patients' code names and identities were not revealed. The evaluation included anterior-posterior and cross-table lateral views of the hips post-operatively on follow-up. The criteria for union and migration by Lakstein was used:

Grade 1: perfect apposition and complete union

Grade 2: $1-3 \mathrm{~cm}$ of displacement (migration)

Grade 3: more than $3 \mathrm{~cm}$ of displacement (non-union)

Grade 4: nonunion requiring a second operation to refix the greater trochanter (complete avulsion plus reoperation). ${ }^{11}$

A trochanteric non-union was defined as the presence of a radiolucent line between the trochanter and displacement of more than $3 \mathrm{~mm}$ from its initial position on follow-up radiographs. ${ }^{10}$ If the radiolucent line was present without migration of the trochanteric fragment, fibrous union in situ was considered. Migration was measured from the tip of the greater trochanter and the shoulder of the femoral component of the AP radiograph. ${ }^{12}$ Agreement between observers (28\%) was quantified by the kappa statistic and was 0.107. Descriptive statistics was used to analyze the measured outcomes. Means were computed for continuous variables and percentages for categorical variables. 


\section{RESULTS}

Out of nine patients screened, two were excluded from the study - one patient did not have radiographs stored in the outpatient department, and another patient did not have outpatient charts for review. Included were two men and five women. The average age of patients at the time of surgery was 52.9 years, (range, 29-74). The average post-op followup was 67 weeks (range, 2-240).

Five were primary surgeries: three for developmental hip dysplasia, one for avascular necrosis, and one for LeggCalve-Perthes disease. Two were revision surgeries, one for replacement of partial hip secondary to protrusio acetabuli and the other for replacement of a total hip for migration of the cemented acetabular component. One patient underwent single-stage replacement for bilateral hips, one patient underwent total knee arthroplasty for the ipsilateral limb 15 months post-hip surgery, and another patient had a subtrochanteric fracture of the contralateral limb that was treated with intramedullary nailing 42 months after the hip surgery.

There were no reported dislocation or revision rates for the set of patients. Hip range of motion was at an average of 42 degrees (range, 40-45) for abduction, 98 degrees (range, 70-120) for flexion. Six patients were ambulatory assisted with a walker (4/6) and cane (2/6); the seventh patient did not have notes on the mode of ambulation on follow-up. There were no reports of pain on the operated side post-op. One patient reported pain on the contralateral hip and knee, and another patient-reported pain on the contralateral hip.

Radiographic evaluation showed both complete union and migration in 36\% and both fibrous union in situ and displacement of more than $3 \mathrm{~cm}$ in $7 \%$.

\section{DISCUSSION}

The modified trochanteric slide osteotomy has been introduced to maximize exposure and optimize the biomechanics of the hip while preserving the posterior capsule to resist posterior dislocation. ${ }^{9}$ The current study reported no dislocation and revision for the small set of patients with the osteotomy. Goodman reported a $14 \%$ dislocation rate in revision cases. ${ }^{9}$ Lakstein reported $4.8 \%$ but did not attribute the dislocation to the osteotomy due to its good healing. ${ }^{10}$ Revisions were for hardware removal for symptomatic bursitis, ${ }^{6,7}$ component migration and infection, ${ }^{6}$ dislocations, ${ }^{13}$ non-union, implant loosening, and femoral fractures. ${ }^{14}$

Lakstein reported persistent trochanteric pain as the most common symptomatic complication at $15.6 \%,{ }^{13}$ but $4.8 \%$ only underwent surgery for hardware removal, which resolved the pain. Similar results were reported by Bal for bursitis, where implants were also removed. ${ }^{7}$ Glassman did not attribute pain to non-union, despite six cases of symptomatic hardware. ${ }^{7}$ The current study is consistent with these findings, despite low rates of radiographic union and no reported cases of pain.

The current study reported range of motion at hip flexion (average $=98$ degrees) and abduction (average $=$ 42 degrees), however, these results cannot be compared to functional outcomes by active and resisted hip abduction, ${ }^{12}$ Trendelenburg sign, ${ }^{6}$ and abductor lurch. ${ }^{6,10,12,13}$

Radiographic evaluation was not comparable to available literature, with only $36 \%$ of reported union and $7 \%$ of nonunion. Union rates of previous studies were $83-92 \%$ and non-union at $3-10 \% .{ }^{6,10,13,14}$ However as previously discussed, the pain was not present despite poor union rates. Patients in the current study also were reported to be ambulatory during follow-up, $85 \%$ assisted by a walker or a cane.

The modified trochanteric slide osteotomy aids in exposure and dislocation for complicated primary hip procedures. Engh preferred the trochanteric slide to protect the abductor mechanism by an osteoporotic or lytic greater trochanter, ${ }^{1}$ which was also recognized by Glassman for procedures that warranted adjustments in leg length, and to expose the acetabulum, proximal femur, and shaft. ${ }^{6}$ Patients included in the study had similar indications and diagnoses and needed a more extensive approach for their procedures.

Limitations of the study include a small data pool, which could yield bias in the results. With the small pool, data available for evaluation were limited as well. Records were assessed retrospectively with no uniform data prospectively prepared for future data gathering, thus the limited outcomes. Radiographs were collected based on availability and might not have been representative of due follow-up. Immediate post-operative plates were not available for the evaluators and were not used as a comparison for the assessment of bony union.

Recommendations for future study include a wider pool of subjects, to include patients done by the supervising investigator in other institutions. Another is to start a prospective study for better data gathering, where variables could be completed and tested for each participant.

\section{CONCLUSION}

Despite the limited data, the modified trochanteric slide approach is a viable option for a subset of patients for exposure and biomechanical advantage for primary and revision hip arthroplasty. The radiographic outcomes are acceptable for asymptomatic and ambulatory patients. Further studies are warranted for more detailed outcomes and results.

\section{Statement of Authorship}

Both authors participated in the data collection and analysis and approved the final version submitted.

\section{Author Disclosure}

Both authors declared no conflicts of interest. 


\section{Funding Source}

This study was self-funded.

\section{REFERENCES}

1. Engh CA. Trochanteric sliding osteotomy hip arthroplasty exposure. Semin Arthroplasty. 2004 Apr; 15(2):119-20.

2. Gross AE, Goodman, SB. Surgical exposures for primary and revision total hip arthroplasty: modified trochanteric slide. Semin Arthroplasty. 2004 Apr; 15(2):122-25.

3. Barrett WP. Standard trochanteric osteotomy. Semin Arthroplasty. 2004 Apr; 15(2):108-12.

4. Nicholson P, Mulcahy D, Fenelon G. Trochanteric union in revision hip arthroplasty. J Arthroplasty. 2001 Jan; 16(1):65-9.

5. Archibeck MJ, Rosenberg AG, Berger RA, Silverton CD. Trochanteric osteotomy and fixation during total hip arthroplasty. J Am Acad Orthop Surg. 2003 May-Jun; 11(3):163-73.

6. Glassman AH. A Technique of extensile exposure for total hip arthroplasty. J Arthroplasty 1987; 2(1):11-21.

7. Bal BS, Kazmier P, Burd T, Aleto T. Anterior trochanteric slide osteotomy for primary total hip arthroplasty. review of nonunion and complications. J Arthroplasty. 2006 Jan; 21(1):59-63.
8. Hadjicostas PT, Thielemann FW. The use of trochanteric slide osteotomy in the treatment of displaced acetabular fractures. Injury. 2008 Aug; 39(8):907-13.

9. Goodman S, Pressman A, Saastamoinen H, Gross A. Modified sliding trochanteric osteotomy in revision total hip arthroplasty. J Arthroplasty. 2004 Dec; 19(8):1039-41.

10. Lakstein D, Backstein DJ, Safir O, Kosashvili Y, Gross AE. Modified trochanteric slide for complex hip arthroplasty: Clinical Outcomes and Complication Rates. J Arthroplasty. 2010 Apr; 25(3):363-8.

11. Menon PC, Griffiths WEG, Hook WE, Higgins B. Trochanteric osteotomy in total hip arthroplasty. J Arthroplasty 1998; 13(1):92-6.

12. Romero AC, Imrie S, Goodman SB. Sliding trochanteric osteotomy preserves favorable abductor biomechanics in revision total hip arthroplasty. J Arthroplasty. 2001 Jan; 16(1):55-64.

13. Lakstein D, Kosashvili Y, Backstein D, Safir O, Gross AE. Trochanteric slide osteotomy on previously osteotomized greater trochanters. Clin Orthop Relat Res. 2010 Jun; 468(6):1630-4.

14. Langlais F, Lambotte JC, Collin P, Langlois F, Fontaine JW, Thomazeau H. Trochanteric slide osteotomy in revision total hip arthroplasty for loosening. J Bone Joint Surg Br. 2003 May; 85(4): 510-6.
The Acta Medica Philippina is now accepting original scientific papers, review articles and case reports for its upcoming issues. Please follow the format for submission as indicated in the "Instructions to Authors" elsewhere in the journal. All papers received shall be properly acknowledged. For inquiries and submission of proposals, please email us at actamedicaphilippina.upm@up.edu.ph 